\title{
Soliton topology versus discrete symmetry in optical lattices
}

\author{
Yaroslav V. Kartashov, ${ }^{1}$ Albert Ferrando, ${ }^{2}$ Alexey A. Egorov, ${ }^{3}$ and Lluis Torner ${ }^{1}$ \\ ${ }^{1}$ ICFO-Institut de Ciencies Fotoniques, Universitat Politecnica de Catalunya, Barcelona, Spain. \\ ${ }^{2}$ Interdisciplinary Modeling Group, Departament d'Òptica, \\ Universitat de València. Dr. Moliner, 50. E-46100 Burjassot (València), Spain. and \\ ${ }^{3}$ Physics Department, M. V. Lomonosov Moscow State University, 119899, Moscow, Russia.
}

(Dated: June 24, 2018)

\begin{abstract}
We address the existence of vortex solitons supported by azimuthally modulated lattices and reveal how the global lattice discrete symmetry has fundamental implications on the possible topological charges of solitons. We set a general "charge rule" using group-theory techniques, which holds for all lattices belonging to a given symmetry group. Focusing in the case of Bessel lattices allows us to derive also a overall stability rule for the allowed vortex solitons.
\end{abstract}

Vortex solitons in nonlinear systems (for a review see 1]) characterized by a discrete symmetry have been numerically predicted in two-dimensional arrays of evanescently coupled waveguides 2], harmonic refractive index gratings imprinted in cubic media [3], as well as in photonic crystal fibers with defects [4]. Because of the imprinted refractive-index modulation, such vortices can be made stable in contrast to their ring-shaped counterparts in uniform focusing media. Recently, vortices having unit topological charge have been experimentally observed in optically-induced lattices in photorefractive media [5]. The very refractive index modulation causing the stabilization of vortex solitons simultaneously imposes restriction on the possible topological charges of the vortices dictated by the finite order of allowed discrete rotations 6]. A corollary of such result is that the maximum charge of stable symmetric vortex in two-dimensional square lattices is one.

However, square lattices are just one particular example of guiding structures accessible for experimental exploration. Another interesting class of such structures with a new global rotational symmetry is constituted by azimuthally modulated lattices, also offering a wealth of new opportunities. For example, in such lattices the order of rotational symmetry may be higher than 4 , in contrast to square lattices, a property that has direct implications in the possible topological charges of symmetric vortex solitons supported by such lattices.

In this Letter we explore the connection existing between the lattice discrete symmetry and the topology of the allowed vortex solitons by means of a general grouptheory approach. We find that azimuthally modulated lattices imprinted in focusing medium can support symmetric vortex solitons carrying phase dislocations with topological indices higher that one. The higher the symmetry order of the lattice, the higher the allowed vortex topological charge. We also address the stability of the allowed vortex soliton families, taking as a particular example the case of azimuthally modulated optically-induced Bessel lattices.

Optical lattice induction in anisotropic nonlinear materials introduced in 7] opens broad prospects for cre- ation of reconfigurable refractive index landscapes with different types of nondiffracting beams, including Bessel beams [8]. Accurate approximations of Bessel beams can be generated experimentally in a number of ways. Known techniques include illumination of annular slit in the focal plane of a lens, conical axicons, as well as more complicated interferometric and holographic techniques (see 9] and references therein). Thus, azimuthally modulated lattices of any desired order can be optically induced by higher-order Bessel beams [8]. Because of the diffractionless nature of Bessel beams, they are to be launched collinearly along the anisotropic nonlinear material (e.g., photorefractive crystal) with a polarization orthogonal to the soliton beams, to make use of incoherent vectorial interactions [7]. The concept can be extended to all relevant physical settings, including Bose-Einstein condensates.

Our starting point is the paraxial nonlinear equation for the complex field amplitude $q$ describing the propagation of light in azimuthally modulated lattice:

$$
i \frac{\partial q}{\partial \xi}=-\frac{1}{2}\left[\frac{\partial^{2}}{\partial \eta^{2}}+\frac{\partial^{2}}{\partial \zeta^{2}}\right] q-q|q|^{2}-p R(\eta, \zeta) q .
$$

The longitudinal $\xi$ and transverse $\eta, \zeta$ coordinates are scaled to the diffraction length and to the input beam width, respectively. The parameter $p$ accounts for the depth of the refractive index profile, whereas the function $R(\eta, \zeta)$ describes the lattice profile. In the particular case of optical lattice induced by higher-order Bessel beam the refractive index profile features beam's intensity distribution $R_{n}(\eta, \zeta)=J_{n}^{2}\left[\left(2 b_{\text {lin }}\right)^{1 / 2} r\right] \cos ^{2}(n \phi)$, where $r=\left(\eta^{2}+\zeta^{2}\right)^{1 / 2}, \phi$ is the azimuth angle, and $b_{\text {lin }}$ is the parameter that sets the transverse lattice scale. In the particular case of optical lattice induction in SBN crystal biased with dc electric field $\sim 10^{5} \mathrm{~V} / \mathrm{m}$, for laser beams with width $10 \mu \mathrm{m}$ the propagation distance $\xi \sim 1$ corresponds to $1 \mathrm{~mm}$ of actual crystal length, while amplitude $q \sim 1$ corresponds to peak intensity about $50 \mathrm{~mW} / \mathrm{cm}^{2}$. The intensity of lattice-creating wave is of the same order and can be varied to tune $p$ in a broad range.

The realization of rotational symmetry in azimuthally 
modulated lattices is different depending on the order of modulation $n$. Lattices of lowest-order with $n=0$ show perfect continuous rotational symmetry since the function $R_{0}(\eta, \zeta)=R_{0}(r)$ has no dependence on $\phi[8]$. In terms of group theory, the symmetry group of a lattice of zero order is $O(2)$. The azimuthal modulation $R_{n}(r, \phi) \sim \cos ^{2}(n \phi)$ changes the symmetry group associated to the rotation transformations. The rotational symmetry group of a lattice of order $n$ is given by the discrete point-symmetry group $\mathcal{C}_{2 n, v}$, corresponding to discrete rotations of angle $\epsilon_{n}=\pi / n$ with respect to a rotation axis perpendicular to the plane and intersecting it at the origin $\left(R_{n}\left(r, \phi+\epsilon_{n}\right)=R_{n}(r, \phi)\right)$ as well as to specular reflections with respect to a number of planes containing the rotation axis 10]. This fact has strong implications in the form of possible symmetric vortex solutions of Eq.(11) $q(\eta, \zeta, \xi)=[u(\eta, \zeta)+i v(\eta, \zeta)] \exp (i b \xi)$, where $u$ and $v$ are real and imaginary parts, respectively, $b$ is a propagation constant, and $m$ describes topological winding number of complex field $q$ that can be defined by the circulation of the gradient of the field phase $\arctan (v / u)$ around the singularity at $\eta, \zeta=0$.

Individual vortices are characterized by a phase singularity, accompanied by a single point of zero amplitude. Discrete rotations are defined with respect to this point, so that all our group theory arguments will apply to individual vortices and not to bound states or clusters of individual vortices owning intrincated phases featuring edges and multiple phase singularities. In this context, individual vortex solutions appear as doubly-degenerated pairs belonging to the two-dimensional representations of $\mathcal{C}_{2 n, v}$ (or of its subgroups) and they are characterized by the index representation $m$. The charge of these solutions is exactly provided by the index representation $m$ [6]. For every value of $m$ one finds a degenerated vortexantivortex pair, with charges $m$ and $-m$, respectively. Since $m$ is limited by symmetry constraints, an upper bound for the values of permitted vortex charges is established if the system enjoys a rotational symmetry of finite order $N$ [6] : $0<m<N / 2$ (for even $N$ ). Since the symmetry group of a lattice of order $n$ is $\mathcal{C}_{2 n, v}$, its symmetry order is $N=2 n$ and thus even. Consequently, we obtain one of the central results of this Letter in the form of a "charge rule" for the allowed values of vortex charges in a lattice of order $n$ :

$$
0<m \leq n-1
$$

We stress that this rule is obtained on the basis of general symmetry arguments and is applicable for a whole class of azimuthally modulated lattices, irrespectively of the details of their local shape and thus method of realization. Some implications of 2 are readily apparent. For example, azimuthally modulated lattices of high orders allow generation of symmetric vortex solitons with topological charges higher than one, in contrast to square lattices.

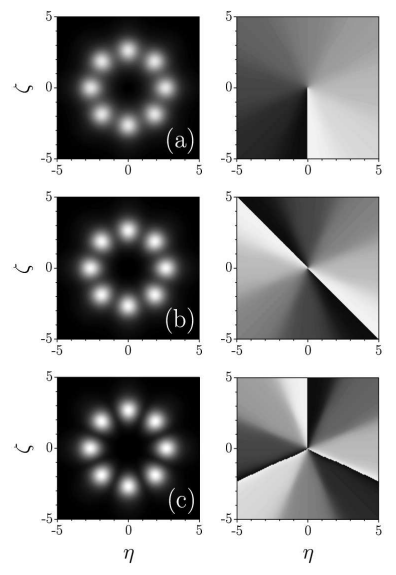

Figure 1: Amplitude (left column) and phase (right column) distributions for the vortex solitons with charges 1 (a), 2 (b), and 3 (c), supported by the fourth-order Bessel lattice at $p=28$. All solitons correspond to $b=1.6$. In left column bright regions correspond to high light intensities, dark regions correspond to low intensities.

We performed a comprehensive numerical analysis of vortex soliton solutions of Eq.(11) for the case of optically induced Bessel lattices to confirm the "charge rule". We searched for symmetric stationary vortex profiles with a relaxation method using as initial guess functions with a phase profile $\exp (i m \phi)$ carrying phase-singularity with charge $m$. The numerical results confirmed the "charge rule" in all cases. A summary of this analysis is presented in Table @ No higher-order vortex solitons have been found to exist above the maximum limit given by Eq. (2). The amplitude and phase distributions for allowed vortices with charges 1, 2 and 3 in a Bessel lattice of fourth order are shown in Fig. 1] The presence of focusing nonlinearity tends to increment the localization effect generating the typical patterns of bright well-localized spots. Notice, that in sufficiently deep lattices at fixed $b$ and $p$ the bright spots in the vortex intensity distribution become more pronounced with increase of vortex charge $m$, while radius of the vortex remains almost unchanged since it is mainly determined by transverse lattice scale. This is in contrast to vortices in uniform medium that broaden notably with increase of $m$ at fixed $b$.

The properties of vortex solitons supported by fourthorder azimuthally modulated Bessel lattice are summarized in Figs. 2(a)-(d). The vortex soliton is characterized by its energy flow $U=\iint|q|^{2} d \eta d \zeta$. The dependence $U(b)$ for a lowest-order vortex soliton with $m=1$ is shown in Fig. 2(a). The different behavior of the $U(b)$ curve for a shallow $(p=4)$ or deep $(p=14)$ lattice indicates the importance of the confining properties of the Bessel lattice. The energy flow is a non-monotonic function of the propagation constant for small lattice depths $(p=4)$ whereas for large values of $p$ (approximately more or equal than 6 ) it monotonically increases with 

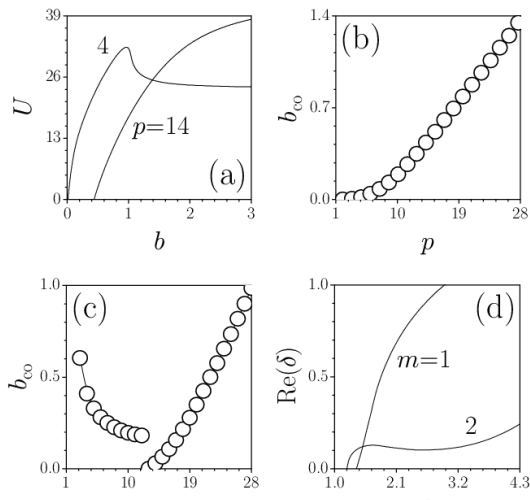

$p$
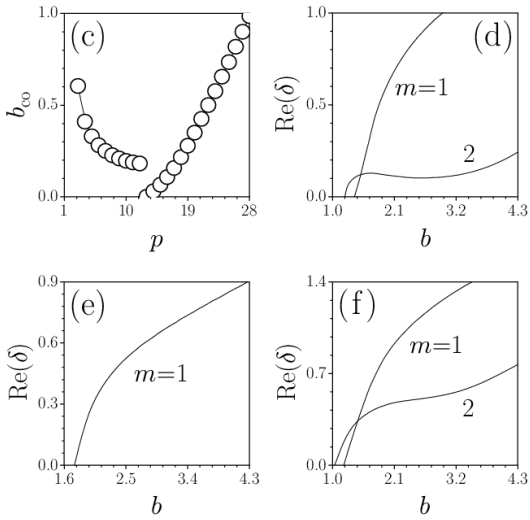

Figure 2: (a) Energy flow $U$ versus propagation constant $b$ for a vortex soliton with $m=1$. Propagation constant cutoff $b_{\text {co }}$ versus lattice depth $p$ for vortex solitons with $m=1$ (b) and $m=3$ (c). (d) Real part of perturbation growth rate $\delta$ versus propagation constant at $p=28$. Panels (a)-(d) correspond to fourth-order Bessel lattice. Real part of perturbation growth rate versus propagation constant for vortex solitons in (e) third- and (f) fifth-order lattices at $p=28$.

$b$. The deeper is the lattice the more pronounced is the azimuthal modulation of the vortex intensity profile.

In contrast, $m=1$ vortices in shallow lattices may undertake an abrupt change of behavior at a critical value of the propagation constant (see the inflection point of the $U(b)$ curve at $p=4)$. With increase of energy flow, bright spots forming such vortices may eventually merge into a single weakly modulated bright ring, since the confining effect of the shallow Bessel lattice is not sufficient to ensure the trapping of light in the higher refractive index regions. At a given value of $p$ the energy flow of the vortex soliton vanishes at certain cutoff $b_{\text {со }}$ on propagation constant (Fig. 2(a)). Close to the cutoff, vortex with charge $m=1$ broadens drastically in shallow lattices, while in deep lattices brigth well-localized spots are always resolvable in vortex intensity profile. As shown in Fig. 2(b), for vortex with charge $m=1$ the cutoff is a monotonically increasing function of the lattice depth $p$. For higher-order vortices with charges $m>1$ the dependence $b_{\text {со }}(p)$ may be discontinuous (Fig. 2(c)). Thus, in shallow lattices higher-order vortices cease to exist in the cut-off without any topological transformation (left branch of the $b_{\text {co }}(p)$ curve), while in deep enough lattices they drastically broaden in the cutoff where vortex energy flow vanishes (right branch).

Next we conducted a detailed linear stability analysis for the allowed vortex soliton families. We searched for perturbed solutions of Eq.(1) in the form $q(\eta, \zeta, \xi)=$ $\left[u(\eta, \zeta)+u_{p}(\eta, \zeta, \xi)+i v(\eta, \zeta)+i v_{p}(\eta, \zeta, \xi)\right] \exp (i b \xi)$, where $u_{p}$ and $v_{p}$ are real and imaginary parts of perturbation, respectively. The linearized evolution equations for perturbation components are

$\frac{\partial u_{p}}{\partial \xi}=-\frac{1}{2} \Delta_{\perp} v_{p}+b v_{p}-\left[2 u v u_{p}+\left(3 v^{2}+u^{2}\right) v_{p}\right]-p R v_{p}$

$-\frac{\partial v_{p}}{\partial \xi}=-\frac{1}{2} \Delta_{\perp} u_{p}+b u_{p}-\left[2 u v v_{p}+\left(3 u^{2}+v^{2}\right) u_{p}\right]-p R u_{p}$

where $\Delta_{\perp}$ stands for the transverse Laplacian. We solved this system numerically by means of a split-step Fourier method with noisy initial conditions to get perturbation profiles and their growth rates.

The results of the stability analysis are summarized in Figs. 2(d)-(f) and in Table \ for lattices with $n$ up to 6. Thus, in fourth- and fifth-order Bessel lattices (Figs. 2(d) and 2(f)), vortices with $m=1$ and 2 were found to be unstable in the entire domain of their existence, while in third-order lattice the unstable vortex carries charge $m=1$ (Fig. 2(e)). Both exponential and oscillatory instabilities are encountered for such vortices. In contrast, third-order lattice can support stable vortex with charge $m=2$, fourth-order lattice supports stable vortex with $m=3$, and fifth-order lattice supports stable vortices with $m=3$ and 4 . In shallow lattices the domains of existence of such vortices feature multiple stability and instability windows, while for deep enough lattices such vortices become stable in the entire domain of their existence. The physical origin of such stabilization is the higher confinement of radiation in regions with higher refractive index that reduces the strength of nonlinear interactions of bright spots forming the vortex. In experiment vortex stabilization would become apparent upon gradual increase of intensity of the lattice-creating beam.

Similar comprehensive linear stability analysis conducted for all lattices with orders $n$ up to 20 with various depths $0<p<100$ enabled us to derive the important "stability rule" for vortex solitons, which may be stable (i.e., a necessary but not sufficient condition) only if the vortex topological charge satisfies the condition

$$
\frac{n}{2}<m \leq n-1
$$

with the exception for $n=2$, when the only existing symmetric vortex with charge $m=1$ may be stable. Similarly to the cases of third-, fourth-, and fifth-order Bessel lattices (Figs. 2(d)-(f)), vortices whose charges satisfy the condition 3 become stable in the entire domain of their existence for deep enough lattices.

Notice that the phase variation $\delta \phi$ between neighboring lattice maxima for vortices with charges 3 verifies $\pi / 2<$ $\delta \phi<\pi$. On intuitive grounds, this difference is consistent with a repulsive interaction between bright spots forming 


\begin{tabular}{|c|c|c|c|c|c|}
\hline Lattice order & \multicolumn{5}{|c|}{ Available charges and stability status } \\
\hline \hline$n=2$ & $\begin{array}{c}m=1 \\
\text { stable }\end{array}$ & & & & \\
\hline$n=3$ & $\begin{array}{c}m=1 \\
\text { unstable }\end{array}$ & $\begin{array}{c}m=2 \\
\text { stable }\end{array}$ & & & \\
\hline$n=4$ & $\begin{array}{c}m=1 \\
\text { unstable }\end{array}$ & $\begin{array}{c}m=2 \\
\text { unstable }\end{array}$ & $\begin{array}{c}m=3 \\
\text { stable }\end{array}$ & & \\
\hline$n=5$ & $\begin{array}{c}m=1 \\
\text { unstable }\end{array}$ & $\begin{array}{c}m=2 \\
\text { unstable }\end{array}$ & $\begin{array}{c}m=3 \\
\text { stable }\end{array}$ & $\begin{array}{l}m=4 \\
\text { stable }\end{array}$ & \\
\hline$n=6$ & $\begin{array}{c}m=1 \\
\text { unstable }\end{array}$ & $\begin{array}{c}m=2 \\
\text { unstable }\end{array}$ & $\begin{array}{c}m=3 \\
\text { unstable }\end{array}$ & $\begin{array}{l}m=4 \\
\text { stable }\end{array}$ & $\begin{array}{l}m=5 \\
\text { stable }\end{array}$ \\
\hline
\end{tabular}

Table I: Table showing the available charges and the stability status of vortex solutions for different lattice orders. The stable status means that it is possible to stabilize the vortex under consideration by a suitable election of the Bessel lattice parameters.
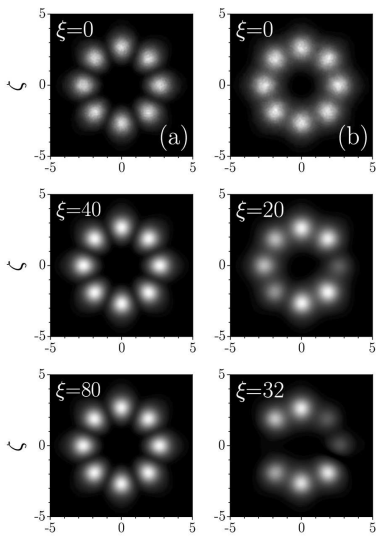

$\eta$

$\eta$

Figure 3: Propagation dynamics of vortex solitons with $m=3$ (a) and $m=1$ (b) supported by fourth-order Bessel lattice in the presence of white input noise with variance $\sigma_{\text {noise }}^{2}=0.01$. Bright regions correspond to high light intensities, dark regions correspond to low intensities. Both solitons correspond to $b=1.6$ and $p=28$.

the vortex (Fig. 11), that are compensated by the lattice and lead to stable vortex propagation, similarly to the case of multipole beams [8].

Direct numerical integration of Eq.(1) with input conditions $q(\eta, \zeta, \xi=0)=w(\eta, \zeta)[1+\rho(\eta, \zeta)]$, where $w(\eta, \zeta)$ is the stationary solution and $\rho(\eta, \zeta)$ is the white noise with variance $\sigma_{\text {noise }}^{2}=0.01$, fully confirmed results of linear stability analysis. In the presence of noisy perturbations, vortices whose charges satisfy the "stability rule" propagate undistorted over hundreds of diffraction lengths, while unstable representatives of vortex soliton families are rapidly destroyed upon propagation (Fig 3 ). The decay of unstable vortex is accompanied by progressive increase of intensity oscillations in neighboring bright spots, until only several spots remain in the output pattern.
In conclusion, we set a general connection between the discrete rotational symmetry of azymuthally modulated lattices and the topological winding number of the allowed symmetric vortex solitons. We derived a "charge rule" and "stability rule"; the "charge rule" is intended to be general for lattices with a given global symmetry order and does not depend on the way in which lattice is created (optical induction in anisotropic nonlinear material or in Bose-Einstein condensates, direct fabrication in the case of photonic crystal, or other methods), while the details of stability of the allowed vortex families are expected to depend on the local properties of the particular lattice considered. The rules predict, for example, that in contrast to square lattices, suitable azimuthally modulated lattices support stable symmetric vortex solitons with higher-order topological charges. Because of the general nature of the geometrical method used in our derivation, we anticipate that a similar charge rule should hold for scalar soliton systems with comparable azymuthal symmetry in a variety of physical settings.

This work was partially supported by contracts BFM2002-2861 and TIC2002-04527-C02-02 from the Government of Spain, and by Generalitat Valenciana (grants GV04B-390 and Grupos03/227).

[1] A. S. Desyatnikov, Y. S. Kivshar, and L. Torner Optical vortices and vortex solitons, Progr. in Opt. vol. 47, 2005. arXiv: nlin.PS/0501026

[2] B. A. Malomed and P. G. Keverkidis, Phys. Rev. E 64, 026601 (2001).

[3] J. Yang and Z. H. Musslimani, Opt. Lett. 28, 2094 (2003); B. Baizakov, B. A. Malomed, and M. Salerno, Europhys. Lett. 63, 642 (2003).

[4] Ferrando et al., Opt. Express 12, 817 (2004).

[5] Neshev et al., Phys. Rev. Lett. 92, 123903 (2004); Fleischer et al., Phys. Rev. Lett. 92, 123904 (2004).

[6] Ferrando et al., Opt. Express 13, 1072 (2005). A. Ferrando, M. Zacarés, and M. A. García-March, arXiv:nlin.PS/0411005 (2004).

[7] Fleischer et al., Nature 422, 147 (2003); Neshev et al., Opt. Lett. 28, 710 (2003); Chen et al., Phys. Rev. Lett. 92, 143902 (2004); Martin et al., Phys. Rev. Lett. 92, 123902 (2004).

[8] Y. Kartashov, V. A. Vysloukh, and L. Torner, Phys. Rev Lett. 93, 093904 (2004); Kartashov et al., J. Opt. B 6, 444 (2004); Phys. Rev. E 70, 065602(R) (2004); Y. V. Kartashov, V. A. Vysloukh, and L. Torner, Phys. Rev. Lett. 94, 043902 (2005).

[9] J. Arlt and K. Dholakia, Opt. Comm. 177, 297 (2000); Garcés-Chávez et al., Nature 419, 145 (2002); D. McGloin, V. Garcés-Chávez, and K. Dholakia, Opt. Lett. 28, 657 (2003).

[10] M. Hamermesh, Group theory and its application to physical problems, Addison-Wesley series in physics (AddisonWesley, Reading, Massachusetts, 1964), 1st ed. 